\title{
Hegemonía cultural del tener frente al ser: expresiones mediáticas y educativas
}

\section{Cultural hegemony of have against being: media and educational expressions}

\author{
Carlos Riádigos Mosquera ${ }^{1}$ \\ carlos.riadigos.mosquera@udc.es \\ Universidade Federal de ViÇosa, Brasil
}

\section{Resumen:}

En sistemas de organización social basados en la acumulación de capital, las diferentes expresiones culturales que surgen dentro de ellos se ven influenciadas por ésta, de forma que lo que se investiga, piensa, cree y educa es mediado por parámetros basados en el lucro. Así, algunos ejes clave sobre los cuales el ser humano articula su pensamiento y acción están en creciente proceso de mutación, progresivamente consolidados sobre un esquema de ideas que en este texto será relacionado con la cultura hegemónica del tener, con potencial vertebrador y que se impone sobre otra basada en el ser, lo que aquí es relacionado con necesidades y expresiones humanas existenciales que van más allá de la acumulación material. A través de la discusión teórica, el objetivo perseguido es repensar cómo mecanismos de aculturación tan importantes como los medios de comunicación o la educación influyen y son influidos por paradigmas de pensamiento profundamente arraigados y relacionados con la propiedad como elemento constituyente, para imaginar la reconstrucción de otros enfocados en ne-

\begin{abstract}
:
In systems of social organisation based on capital accumulation, the different cultural expressions arising from them are influenced by capital accumulation. Consequently, what we research, think, believe and educate is mediated through paremeters based on profit. So, some key pillars on which human beings articulate their thinking and actions are subject to an increasing process of change, gradually consolidated on an scheme of ideas that in this text will be linked to the dominant culture of having, with structuring potential and imposed over another form of culture based on being, what here is related to existential human needs and expressions that go beyond material accumulation. Through theoretical discussion, the aim of this study is to rethink how acculturation mechanisms as important as media or education influence and are influenced by paradigms of thinking which are deeply rooted and related to property as a constituent element. In doing so, we seek to imagine the reconstruction of another form of thinking focused on a wide understanding of human needs and related to intangible and social aspects.
\end{abstract}

1 Dirección para correspondencia (correspondence address):

Carlos Riádigos Mosquera. Programa de Pós-GraduaÇão. Universidade Federal de ViÇosa. Rúa Isolina, 441, apartamento 301. 20710-070 Río de Janeiro, Brasil. 
cesidades humanas más amplias y relacionadas con aspectos inmateriales y sociales.

\section{Palabras clave:}

Cultura; hegemonía; tener; ser; medios; educación.

\section{Keywords:}

Culture; hegemony; having; being; media; education.

\section{Résumé:}

Dans les systèmes d'organisation sociale fondés sur l'accumulation de capital, les différentes expressions culturelles qui y apparaissent sont influencées par celle-ci, de sorte que ce qui est recherché, penser, créer et éduquer sert de médiateur par les paramètres basés sur le profit. Donc, certains fondements essentiels sur lesquels les êtres humains articulent leur réflexion et l'action sont des processus de mutation, progressivement consolidés sur un aperçu des idées qui dans ce texte seront liés à la culture dominante d'avoir, avec un potentiel de structuration et qui s'impose sur une autre basé sur l'être, si cela est lié à des expressions humaines et aux besoins existentiels qui vont au-delà de l'accumulation de matière. Par la discussion théorique, le but est de repenser la façon dont les mécanismes d'acculturation si importants comme les médias de communication ou de l'éducation influencent et sont influencés par les paradigmes de pensée profondément enracinés et liés à la propriété comme un élément constitutif, d'imaginer la reconstruction d'autres mécanismes axés sur de plus amples besoins humains et liés aux aspects sociaux et matériels.

\section{Mots clés:}

La culture; I’hégémonie; avoir; être; les médias; I'éducation.

Fecha de recepción: 13-6-2015

Fecha de aceptación: 4-2-2016

\section{Introducción}

Todo pensamiento humano está condicionado por su finitud, una limitación temporal consustancial a nuestra propia naturaleza. Lo que en distintos momentos y lugares fue asumido como lógico, fundamental y permanente, en otros pudo haber sido desconsiderado o cambiado con el paso del tiempo. En la actualidad, la enorme importancia del tener en gran parte de las sociedades del planeta (Fromm, 2007) tiene la capacidad de mediar todo tipo de comportamientos tanto a nivel individual como colectivo, en detrimento de otros más relacionados con experiencias no directamente vinculadas con la posesión de bienes materiales, más cercanas a ser. Para entender el por qué de estos cambios temporales y espaciales, se hace necesario recurrir a causas primarias de generación ideológica, para lo el concepto de hegemonía cultural puede ser de gran ayuda, referido al modo que la clase dominante, fuertemente 
relacionada en contextos capitalistas con la acumulación de capital, tiene para poder marcar la norma o el sentido común, a través de una serie de percepciones, explicaciones, valores y creencias (Gramsci, 2010), lo que es una forma de dominación aparentemente inocua, limpia y neutra.

La estructuración del pensamiento entorno al tener bebe, entre otras fuentes, de la lógica liberal en la que la propiedad conforma una parte indispensable de la base cultural para la legitimación de los derechos básicos individuales. Según esta forma de concebir el mundo, las personas son lo que son con respecto a las demás en buena medida en función de aquello que puedan poseer, lo que puedan tener, y siempre desde la óptica individual y/o familiar, ya que la posesión de bienes pública y/o colectiva está alejada del núcleo ideológico de este paradigma. El dominio de la cultura que entiende las relaciones sociales principalmente como potenciales herramientas para la posesión de bienes, y que silencia capacidades y posibilidades de construcción colectiva de un mundo basado en otro tipo de necesidades humanas y ambientales, se basa en el individuo autosuficiente e independiente como marco primario de referencia política, económica y social (Beck y Beck-Gernsheim, 2003), fenómeno intensificado y diversificado con la corriente neoliberal. De esta forma, se viene produciendo una progresiva traslación de formas más "moleculares" de entender la sociedad a otras más "atómicas", con la consiguiente pérdida de referencias comunales y con la individualidad enfocada hacia la propiedad como el más fundamental de los derechos.

Es intención de este texto confrontar lo anterior con la lógica del ser, aquella que conduce a priorizar ingredientes para la existencia humana venidos desde posiciones más ecológicas en lo intelectual, social y ambiental, a través de la valorización de facetas inmateriales de la vida y que habitualmente son relegadas a un segundo plano, como la ética, la moral, la espiritualidad, o las relaciones comunitarias y con el medioambiente. Esta concepción requiere volver a considerar que la vida sencilla y en comunidad son valores a redescubrir y se enriquecen cuando se cede un poco de libertad individual para apostar por la equidad en derechos y recursos sociales.

Son modelos muy diferentes, y que demandan de la educación papeles casi antagónicos, al mismo tiempo que pueden dejar entrever hacia qué tipo de sociedad se apuesta desde las instituciones que regulan las políticas educativas. Los intentos en los últimos años de introducir asignaturas tan contrapuestas como "Educación Financiera" y "Educación 
para la Ciudadanía" en el Estado Español, deja clara la existencia de al menos dos caminos no complementarios a la hora de entender la sociedad y el papel de la educación en ella, o lo que puede ser equivalente, la diferencia entre una perspectiva anclada en el tener o en el ser.

\section{Dominio del tener y obediencia del ser}

Dentro del marco conceptual de la hegemonía cultural, un importante elemento para entender la prevalencia ideológica de cada período es la comprensión del corpus teórico que la sostiene, detrás del cual debe haber un grupo de intelectuales que les doten de legitimidad y lo empleen. La religión fue durante siglos la principal herramienta utilizada para estos fines, sustituida paulatinamente a partir de la Ilustración por la ciencia moderna, actualmente aliada con la lógica productivista (Santos, 2007). El tipo de investigación que marca las pautas en el contexto capitalista es la que responde eficazmente a sus demandas, al tiempo que se produce una variada segregación de aquella progresista y promotora de cambios en relación al desequilibrio de fuerzas existente a la hora de investigar y conocer. Esto provoca la falta de empoderamiento de una forma de conocer más ecológica en las universidades, debido por ejemplo a la pobre financiación, silenciando aquello que no es prosistema (Giroux, 2005). Ese silenciamiento se relaciona con prácticas de investigación que utilizan diferentes modalidades de discurso, como concentrarse en lo superficial sin abordar lo estructural, justificar las realidades negativas como autogeneradas y sin responsabilidades sociales, psicologizar los problemas, o convencer de la imposibilidad del cambio en un momento concreto (Torres Santomé, 2011). Un claro ejemplo de esto es la asimilación por parte del gobierno de los Estados Unidos en la década de los años 80 de los preceptos económicos monetaristas de la Ilamada "Escuela de Chicago", con Milton Friedman como figura destacada, basados en la liberalización del comercio, la total libertad para los mercados, acabar con la inflación y privatizar el sector público. Principios e ideas que se tornaron hegemónicas a través de su uso e implementación con el "Consenso de Washington" del año 1989, por ejemplo, un conjunto de políticas económicas acordadas fundamentalmente por organismos financieros internacionales y centros económicos con sede 
en Washington D.C., sobre lo que consideraban que sería la mejor forma de desarrollo económico para los países latinoamericanos (Klein, 2010).

La ciencia es utilizada para dotar de legitimación a toda posición de poder, a través de la elevación de su conocimiento al estatus de científico. Con ello se puede justificar desde posiciones consideradas como objetivas y neutras diferentes injusticias sociales, por ejemplo, a través de herramientas como la economía entendida como ciencia aséptica y no ligada con las intenciones humanas (Max Neef y Smith, 2014), o la utilización de las ciencias sociales desde una perspectiva tecnocrática (Ema, 2013). En esta línea, también se pueden dar procesos de falsa objetivación relacionados con las prácticas divisorias (Foucault, 1988), por las cuales el sujeto es dividido tanto en su interior como con respecto al resto, con contraposiciones como loco-cuerdo, enfermo-sano, criminal-inocente, dicotomías muy vinculadas a la ciencia moderna (Santos, 2007) y con el poder de decidir qué o quién está dentro o fuera de la normalidad según los parámetros culturales hegemónicos. Son conceptos que, si bien son habitualmente dominados por quien tiene medios para su control, están en permanente disputa. En el actual contexto de hegemonía neoliberal, mientras para la clase político-económica "criminal" puede ser una persona que protesta, un "antisistema", para la ciudadanía que lucha por democracia entendida desde la justicia social ese término podría hacer referencia a quien, por su afán de tener y acumular, empobrece los derechos elementales de buena parte de la ciudadanía.

Esas herramientas de hegemonía ideológica son utilizadas para debilitar procesos emancipadores y realmente democráticos, bajo la argumentación de que estos empobrecen tradiciones culturales asentadas, por menoscabar valores como los religiosos, patriarcales o patrióticos (Apple, 2002). Esa cultura alternativa sería así un problema por su cuestionamiento del orden social establecido, orden que puede ser dibujado como el de los hombres, blancos, adultos, urbanos, trabajadores, católicos, de clase media, heterosexuales, delgados, sanos y fuertes (Torres Santomé, 2011, p. 225), y que silencia a las culturas con características diferentes y modelos de convivencia alternativos. En este sentido, la raza blanca se configura todavía como eje vertebrador dentro de la cultura dominante (Kincheloe y Steinberg, 1999), por lo que el control del pensamiento de la blancura, con frecuencia bien respaldado por acciones bélicas, relaciona la razón de un grupo con la idea de razón platónica, otorgando el estatus de natural a la blanca y masculina. Por tanto, lo que 
no entra dentro de este esquema no sería puramente razonable y tendría más posibilidades de ser caótico, convirtiendo a la imposición cultural en salvadora, por el componente supuestamente emocional e irracional de los pueblos y razas no alineadas con el "desarrollo". Es algo que va de la mano junto con procesos como el chauvinismo (Nussbaum, 2001), o sea, asumir un punto de vista como el mejor posible, y tratar de modelar la cultura ajena a la propia.

Todo lo anterior, en relación a la cultura del tener, se puede apreciar con lo que viene agravándose desde los años '80 en el contexto estadounidense con respecto a la juventud de raza negra, excluida de las decisiones trascendentales de la sociedad y reprimida si reclama por ello mediante la policía, las cortes penales o las prisiones, y reforzado con la renacida cultura de la seguridad, en detrimento de las libertades civiles, a partir del 11-S de 2001. Lo sucedido en 2015 en Baltimore, Estados Unidos, con el asesinato de varias personas negras a manos de la policía y las posteriores revueltas y enfrentamientos violentos, es otra de las muestras de cómo la hegemonía cultural, relacionada con la blancura y un buen estatus socioeconómico, puede derivar en el uso de la violencia por parte de los instituciones estatales para controlar la diferencia disidente.

El espacio de mejora de la convivencia social generado por décadas del Estado de Bienestar, con conquistas de género, raciales, sexuales, políticas, etc., es sustituido paulatinamente por un énfasis cada vez mayor en la vigilancia, la represión y el control. Lo relacionado con la "seguridad" se convierte poco a poco en negocios altamente lucrativos, dejando por ejemplo el sistema penitenciario o de control aeroportuario en manos privadas. Como sucede con la guerra, al convertir el miedo social en negocio corporativo, son necesarios clientes para el sistema, creciendo sin parar año tras año el número de personas presas en los Estados Unidos. El tener, la generación de beneficio empresarial para esa minoría que ejerce la hegemonía cultural tan ligada a la económica, se impone sobre el ser para la mayoría de la población, la posibilidad de desarrollar aspectos vitales y derechos sociales que vayan más allá de la acumulación de bienes.

Dada la suposición entre neoliberales radicales de que los valores del mercado son más importantes que los valores vinculados a la confianza, compasión, y solidaridad, no sorprende que Wall Street, donde se efectúan 
las ganancias, vea el crecimiento en la industria carcelaria y el creciente confinamiento de jóvenes como una buena noticia. (Giroux, 2005, p. 75)

La cultura del tener y su dependencia del lucro empresarial tiene como protagonista principal a todo lo que envuelve al mundo del consumo, e incluso la infancia no escapa a esta lógica. Niñas y niños son, cada vez más, objeto de interés de las grandes multinacionales para influir en su consumo propio y en el de los adultos, para lo que se utiliza el trabajo de profesionales especialistas en psicología, neurología, pedagogía, etc., al servicio de las empresas (Schor, 2006). Se alía con esto la cultura de las marcas que, a través de sus logos, invaden espacios públicos y con ello nuestras mentes (Klein, 2000). Por lo tanto, se da una actividad parasitaria de ese espacio público para fines privados, similar a la "absorción" por parte de la cultura hegemónica neoliberal y a través de la mercantilización, de reivindicaciones sociales y culturales tales como el feminismo, la homosexualidad, el punk o el hip hop, convirtiendo luchas sociales en promoción de las marcas, a través de la publicidad, algo en lo que Coca Cola es especialista. También es sintomática la degradación ejercida sobre culturas que emergen apartadas de la dominante, por ejemplo la del heavy metal, el rap o el porno, por ser para la hegemonía desviaciones culturales producto de la frustración y por no adaptarse a la lógica del mercado (Dijk, 1999).

Esta forma de utilizar la cultura tiene sus efectos directos en el principal pilar de los sistemas culturales, la educación. Contribuye a reforzar la lógica de las escuelas vistas como instituciones disciplinarias que preparan al alumnado para el lugar de trabajo y para consumir, en las que la estandarización es la norma que facilita el dominio del eje conformado por trabajo-consumo-disciplina. Pero además, con la creciente complicidad del sector público con las demandas corporativas, se llega a situaciones en las que, más allá del progresivo debilitamiento de la educación pública mediante políticas de corte neoliberal, cada vez es más frecuente que dirección y profesorado en las escuelas participen en programas promovidos por éstas para conseguir medios extraordinarios para las aulas, habitualmente negados por los presupuestos estatales, permitiendo introducirse en la vida escolar a través de la mezcla de la publicidad con los contenidos pedagógicos, por ejemplo. Junto a la publicidad en textos y espacios escolares y públicos, existen otras formas mediante las cuales los intereses privados invaden este terreno, como la comercialización 
(hacia, en y de las escuelas) (Molnar, 1999) y el mercantilismo (patrocinio de programas y actividades, acuerdos de exclusividad, programas de incentivos, apropiación de espacios, materiales educativos patrocinados, comercialización electrónica, privatización, y recaudación de fondos) (Molnar y Boninger, 2007), conformando así una serie de estrategias mediante las cuales los principales lugares de encuentro educativo público ceden espacio en su lógica del ser, lo que urge revalorizar lo inmaterial y lo que nos debemos unas personas a otras como seres que comparten la misma condición humana (Scanlon, 2003).

\section{Mecanismos para asegurar la hegemonía cultural del ideario de la propiedad privada}

Realidades como las anteriormente narradas no son fortuitas o accidentales, son producto de procesos sociohistóricos mediados por la interacción entre el dominio y la obediencia cultural y política. La cultura del tener ancla sus raíces en el mismo inicio de la vida en comunidad del ser humano, acompañándolo en mayor o menor intensidad durante milenios de evolución y con diferentes intensidades, dependiendo siempre del contexto territorial, cultural, económico, político, militar, etc. de cada momento. Actualmente, la exacerbación de esta forma de entender la vida se nutre del liberalismo clásico y el fortalecimiento de la propiedad privada como forma crecientemente hegemónica de articulación social, corriente por la que no sólo un reducido grupo dominante podía poseer, sino que esto se convirtió en algo un poco más transversal abarcando a personas con derechos de ciudadanía, principalmente a la creciente burguesía.

El desarrollo burgués influyó y se dejó influir por la obra de autores imprescindibles para la expansión de las ideas asociadas a la propiedad privada. Adam Smith (1776) consideraba que gracias al egoísmo particular se logra el bienestar general, de modo que la suma satisfactoria de las acumulaciones individuales podría identificar si una sociedad está en la buena dirección o no. Desde esta perspectiva, el Estado estaría limitado a garantizar el libre mercado y el crecimiento económico, potenciador de esas acumulaciones, y la "mano invisible" del mismo se encargaría de mantener la paz y el equilibrio social.

Esta serie de nociones sobre la vital importancia de la propiedad pri- 
vada conformaban y conforman la base del pensamiento de quien más poder tiene en el contexto capitalista, o sea, quien domina los medios de producción. La evolución contemporánea de esto se traduce en diversificadas facetas, cobrando en nuestros días una importancia fundamental el control cultural y de la información, lo que se expresa en el dominio económico y/o ideológico de los medios de comunicación y la educación. En el caso de los medios y editoriales, un reducido número de familias con enorme poder económico y político controla una gran parte de estos en diferentes lugares. Así, Murdock, Redstone o McGraw (Estados Unidos), Rothermere (Inglaterra), Lagardére o Seydoux (Francia), Li (China), Frías (Brasil), Slim (México), Springer (Alemania), Berlusconi (Italia) o Lara y Polanco (España) (Campos Freire, 2007), entre muchas otras familias, controlan la posesión de estos medios de comunicación y diferentes editoriales de libros de texto escolares, haciéndose responsables así de una parte fundamental de la elaboración cultural de sus respectivas sociedades, produciendo hegemonía.

Como negocio empresarial, tanto la posesión de los medios y editoriales de libros texto como el tratamiento de la información contenida, siguen el patrón de control del tener sobre el ser. Por ejemplo, el derecho de propiedad privada de los medios y su libertad asociada para poder utilizarlos principalmente para generar beneficios (aunque la calidad informativa se vea perjudicada), prima sobre aquel que hace referencia a tener información rigurosa, independiente, democrática y con mayor influencia de lo inmaterial, necesario para llevar una vida en sociedad más diversa, justa y feliz, que vaya más allá de la propiedad, hacia el ser. Esa forma de entender la cultura como propiedad privada favorece el inmovilismo y la falta de diálogo social para su apertura al debate y la mejora, apuntalando la hegemonía sociocultural del momento:

La función de los medios de comunicación de masas es la de divertir, entretener e informar, así como inculcar a los individuos los valores, las creencias y los códigos de comportamiento que les harán integrarse en las estructuras institucionales de la sociedad. (Chomsky, 1990, p. 21)

Esta afirmación de Noam Chomsky tiene más de 20 años y, a pesar del paso del tiempo, parece que lo fundamental de ella poco ha cambiado. La alianza neoconservadora y neoliberal (Apple, 2002) es suficiente para controlar a nivel económico e ideológico la mayoría de medios y 
editoriales de gran alcance al público en buena parte de las democracias representativas occidentales. La obtención del máximo beneficio posible por parte de estas macro empresas de comunicación es, a través de la publicidad y sin temor a ser mostrado, su objetivo prioritario (Serrano, 2012), por encima de tantos otros que podrían tener y que atendiesen a urgencias democráticas y de justicia social.

El control de la opinión pública es un pilar clave para esta forma de entender la comunicación e información, y a la hora de legitimar la idea de la mutua necesidad en el dualismo democracia-capitalismo. La poca diversidad en la posesión de los medios, y por lo tanto de los discursos, perpetúa el dominio del pensamiento único (Ramonet, 1995), pues con demasiada frecuencia las diferencias entre los grupos que tienen cabida en esos medios son superficiales y no abordan debates de fondo. Así, no es posible encontrar grandes medios de comunicación abiertamente anticapitalistas o que den cabida a pensamiento marcadamente contrahegemónico, por ejemplo.

Las formas contemporáneas de control se asocian con las más tradicionales para asegurar el mantenimiento del ordenamiento social. Hannah Arendt (1998) dejó un legado fundamental para entender cómo élites y autócratas operan para conservar un sistema totalitario del que beneficiarse, lo que se perpetúa en buena medida en el actual contexto neoliberal global, mostrando conexiones con sistemas autoritarios en el pasado y su incompatibilidad con sociedades realmente democráticas, por lo que es interesante pensar algunos de esos puntos en común:

- En un sistema totalitario, la destrucción de la diversidad social es crucial, con el propósito de generar masas homogeneizadas, de forma que la dominación sólo sea posible donde éstas existan, siendo su característica principal la atomización, el aislamiento y la soledad. Así, movimientos sociales radicalmente pacíficos como el $15 \mathrm{M}$ o Occupy Wall Street, fueron y aún son perseguidos, golpeados y criminalizados mediante amenazas desde cargos políticos, agresiones policiales o detenciones ilegales, condenados precisamente por la diversidad de su pensamiento y formas, opuestas a las hegemónica;

- Repudio de la población contra todo lo conseguido a nivel institucional, político e intelectual, por el ataque desde el poder a los valores más democráticos de esos sistemas, entregándose la legitimidad de acción y pensamiento a un "salvador", que es quien 
provoca ese distanciamiento. La continuada desconexión de la ciudadanía con respecto a la política en el contexto hegemónico capitalista es una muestra de esto, con precarios procesos electorales cada cuatro años en los que se resume la participación, lo que provoca una lógica desconexión humana e ideológica de las personas con el mundo, y la identificación del término "política" sólo con un grupo de representantes electos;

- La propaganda del sistema permite la socialización y educación favorables al mismo, creando un mundo de ficción en el que quien representa el poder en ese momento se autodefine como "bueno" y quien lo amenaza es considerado como "malo", con la clara finalidad de escribir una historia a medida. Así, la propaganda tendrá éxito en poblaciones subordinadas donde la capacidad crítica y la autonomía de pensamiento hayan perdido validez. Son varios los puntos en los que podría resumirse la propaganda de las sociedades neoliberales para intentar imponer su sentido común y mantener el statu quo: a) el consumo se presenta como medio para conseguir la libertad social; b) el sistema bipartidista se presenta como medio para conseguir la libertad política; c) el sistema de libre mercado se presenta como medio para conseguir la libertad económica; d) el sistema cultural "hollywoodiense" se presenta como medio para conseguir la libertad cultural. Todos ellos sustentados y apoyados por un sistema educativo influenciado hacia estos aprendizajes y un sistema jurídico que asegura su mantenimiento;

- La violencia y terror son otros dos elementos básicos para el totalitarismo. El terror induce al convencimiento de la propaganda, y en el caso de no obtener los resultados deseados se recurre a la violencia para cumplir la doble función de demostración de la supuesta verdad y de castigo frente a la desviación de las normas y leyes. La demonización informativa de líderes y sistemas democráticos de países con grandes cuotas de participación política ciudadana y alternativas económicas al modelo neoliberal, como por ejemplo Bolivia o Ecuador en la actualidad, dan buena cuenta de esto;

- El poder siempre es controlado por el centro, por quien lidera dando órdenes a las diversas capas de alrededor, siendo la infalibilidad de este poder uno de los componentes básicos de la pro- 
paganda. La lealtad total es la base psicológica de la dominación, buscando esto en cada persona y en todas sus esferas vitales. Se produce primero en el ámbito jurídico, situando fuera de la ley a determinados grupos de personas contrarias al régimen, de forma que es mucho más fácil que tenga problemas de tipo legal un movimiento social anticapitalista que uno conservador, por ejemplo.

Continuando con el análisis de procedimientos de aculturación hegemónica, una herramienta imprescindible para entender cómo operan los grandes medios de comunicación corporativos es la que elaboró Sylvain Timsit (2002) con sus 10 Stratégies de Manipulation, habitualmente atribuidas por error a Noam Chomsky, y que sí se complementan con las que anteriormente este autor y Edward S. Herman (1990) recogieron en relación al modelo propagandístico que es utilizado para filtrar la información. Según Timsit, el poder del capital se sirve de esas estrategias en los medios para seguir ejerciendo como tal, orientando a las audiencias en las formas que su trabajo recoge. Pocos ejemplos pueden ilustrar tan bien la situación actual de los medios y estas estrategias como el de la llamada "crisis" en la que están muchos estados europeos, siendo ese calificativo el primero de los intentos de manipulación. Este período en el que estamos aporta numerosas situaciones, en este caso dentro del Estado Español, que pueden ejemplificar esas diez estrategias. Al mismo tiempo, y con respecto a la educación, mucho de lo aquí recogido será relacionado con su expresión en diferentes realidades educativas, especialmente en libros de texto que, con demasiada frecuencia, muestran una marcada tendencia hacia el pensamiento hegemónico (Riádigos Mosquera, 2015):

1. La estrategia de la distracción: por la que se desvía la atención del público de los problemas importantes mediante continuas informaciones superficiales. El conocimiento realmente valioso es relegado a secciones en los peores lugares u horarios, mientras contenidos de deportes, consumo, información rosa o política partidista ocupan los mayores espacios. Actualmente, la información sobre la estafa económica que están sufriendo numerosos países en el contexto "crisis", es enmascarada bajo informaciones técnico-económicas vacías de significado, tales como las referidas a la "prima de riesgo" o los índices Ibex o Down Jones. Así, es posible ver con frecuencia cómo el conocimiento verdaderamente signi- 
ficativo para el alumnado, el más relacionado con su vida diaria como por ejemplo sobre los efectos de la "crisis", es dejado de lado para cumplir con requerimientos de evaluaciones como PISA y pruebas como las de acceso a la universidad, centradas en una lógica continuista y de inmediatez de resultados.

2. Crear problemas y después ofrecer soluciones: que interesan a los grupos hegemónicos y que no serían aceptadas sin esos problemas previos. Por ejemplo, crear o facilitar una crisis económica (2008) o un atentado (11-S) con repercusiones internacionales, para forzar a aceptar como un mal necesario el retroceso de los derechos sociales y el desmantelamiento de los servicios públicos (Klein, 2010), incluido el educativo: para avanzar en la privatización, primero se debilita lo público a través de la falta de recursos, entre otras estrategias, para legitimar así la necesidad de la llegada de la iniciativa privada como salvadora de la situación.

3. La estrategia de la gradualidad: para que se acepten medidas previamente impensables sin provocar una revolución, éstas se aplican gradualmente. Así se imponen privatizaciones, precariedad, flexibilidad o desempleo sin estallido social, siendo el "copago" sanitario o la reforma laboral del 2012 dos ejemplos de esto. En una línea similar, el aumento de la mercantilización escolar o los crecientes intentos de privatización en el sistema educativo son medidas que se intentan implementar poco a poco y siempre con la complicidad de las dos estrategias anteriores en relación a lo público.

4. La estrategia de diferir: para la aceptación de algo impopular también se propone su implantación en el futuro, por ser más fácil aceptar eso que algo inmediato. Se puede apreciar esto con medidas como la ampliación progresiva de la edad para la jubilación de 2011, justificada sobre el recurrente argumento de que el sistema no es "sostenible" (al mismo tiempo que se permiten amnistías o paraísos fiscales). Medidas que entran en vigor progresivamente, anestesiando parcialmente el rechazo a las mismas, y que se complementan a nivel educativo con las estrategias previas y el debilitamiento de lo público.

5. Dirigirse al público como criaturas de corta edad: mensajes y publicidad que utilizan discursos, argumentos, personajes y entonación particularmente infantilizantes para generar respuestas simi- 
lares, sin sentido crítico. La situación política, social y económica actual es habitualmente tratada desde análisis simplistas, falta de rigor y de profundidad, frecuentemente acompañado de una espectacularización de la actividad política que se centra en la confrontación personal, eludiendo abordar ideas de calado estructural. Prácticas de infantilización (Torres Santomé, 2011) son cada vez más comunes en escuelas y libros, dilatando progresivamente las etapas de desarrollo psicológico por la ausencia de abordaje de temáticas de importancia decisiva en el currículo y que faciliten su maduración intelectual.

6. Utilizar el aspecto emocional mucho más que la reflexión: que provoca un uso precario del análisis racional, y por lo tanto del sentido crítico. También, la utilización de las emociones permite abrir el inconsciente para implantar o inyectar ideas, deseos, miedos y temores. En esta dirección, los desahucios, falta de atención sanitaria o hambre en el Estado Español, lejos de ser tratados como un asunto político, son abordados desde las emociones de las personas afectadas, para conseguir mayores índices audiencia y convirtiéndolos en "desgracias inevitables" sin causas políticas. Algo similar sucede cuando se piensa en contenidos curriculares que, a pesar de ser explicitados con frecuencia en clave crítica, se practican después desde una falta de conexión alarmante con problemas políticos, estructurales y desde una perspectiva individual y emocional.

7. Mantener al público en la ignorancia y la mediocridad: siendo incapaz de comprender los métodos utilizados para su control. La calidad de la formación dada al grueso de la población debe ser de mínimos y orientada hacia la producción. Sistemas educativos centrados en la adquisición de habilidades técnicas y cada vez más pobres en lo relacionado con el desarrollo humano o para la comprensión sociopolítica facilitan el adoctrinamiento y la imposición cultural.

8. Estimular al público a ser complaciente con la mediocridad: haciendo creer por ejemplo que la ignorancia o la falta de compromiso están de moda y son algo bueno, lo que se puede ver en el enorme espacio ocupado por los debates televisivos o noticiarios en los que personas sin preparación y casi siempre de la corriente de pensamiento hegemónico copan el tiempo de programación 
para "analizar" la situación económica. Políticas educativas favorecedoras de la precarización en la formación y condiciones laborales del profesorado, por ejemplo, contribuyen en esta dirección (Torres Santomé, 2009).

9. Reforzar la autoculpabilidad: haciendo creer a la mayoría de las personas que son las únicas culpables de su propia desgracia, por no ser aptas o no esforzarse, evitando así la crítica al sistema, estrategia que se pudo apreciar nítidamente con la insistente repetición durante la "crisis" de la frase que decía que en el pasado "vivimos por encima de nuestras posibilidades", que refuerza la culpabilización de las víctimas y la victimización de los culpables. El gran expolio de dinero público en beneficio de los bancos, uno de los motivos por los cuales los países están endeudados, apenas es motivo de debate, y sí el deseo de las familias de comprar una casa o viajar cuando podían acceder al crédito. En este marco de pensamiento, la población tendría los sistemas educativos que "se merece", siendo sus carencias y debilidades únicamente atribuidas a las personas pertenecientes a las comunidades educativas, que no sabrían realizar bien su trabajo.

10. Conocer a los individuos mejor de lo que ellos mismos se conocen: gracias a los avances científicos y tecnológicos, para poder ejercer un mayor control individual. La investigación psicológica y sociológica sobre las personas que posee una minoría social, unido a los enormes avances tecnológicos de empresas como Google o Facebook las cuales, almacenando una ingente cantidad de datos sobre nuestra vida y con finalidades publicitarias y comerciales, facilitan el profundo conocimiento de las personas mediante estudios meticulosos, con la clara finalidad de los beneficios económicos y la previsión del comportamiento social. La insistencia en la psicometría como herramienta para conocer mejor al alumnado camina en este sentido.

Se hace necesario que el dibujo recién creado sea complementado con las nuevas herramientas de nuestra era. Si bien todavía son la televisión, radio, prensa y libros las formas de aculturación dominantes, internet comienza a jugar un papel importante. Además de las inmensas posibilidades emancipadoras que ofrece por su tipo de (no)estructuración, horizontal y en red, ésta es también utilizada para favorecer una 
forma de comprender el mundo fundamentada en la hegemonía cultural occidental y del capital. Los incesantes intentos por limitar la libertad de expresión en la red (Amadeu da Silveira, 2009), siempre en nombre de la seguridad, anteceden al desarrollo de prácticas antidemocráticas que tienen como finalidad obtener información, por la vía que sea, que permita continuar con la hegemonía establecida. En este sentido es significativo el caso de Edward Snowden, antiguo empleado de la National Security Agency (NSA) estadounidense, que está en busca y captura por parte del gobierno de Estados Unidos por revelar información reservada que demuestra la continua violación de todo tipo de Derechos Humanos por parte de esa administración en todo el mundo, y que al mismo tiempo es considerado como un héroe por movimientos sociales contrahegemónicos, lo que deja de manifiesto la lucha abierta dentro de la dicotomía tener-ser. También, la persecución que se hace por ejemplo de las descargas mal Ilamadas "piratas", directamente vinculada a la presión que ejercen sobre diferentes gobiernos los grandes lobbies del entretenimiento cultural, o el reciente intento de diferentes gobiernos para anular la neutralidad de la red y su carácter universal mediante la instauración de un "internet de dos velocidades", son ejemplos de cómo la cultura de la propiedad (vinculada con el Copyright) ejerce mucho mayor poder de decisión que otra más centrada en principios de colaboración social (asociada al Copyleft o Creative Commons).

La hegemonía del tener se muestra así a través de los principales canales de difusión cultural, con la educación como elemento clave en esta ecuación. El aprendizaje de habilidades "útiles" para las necesidades concretas de este tipo de sociedades condiciona fuertemente las agendas educativas, por ejemplo a través de instituciones como la OCDE y su informe PISA, centrado en evaluar la comprensión lectora, la alfabetización matemática y la alfabetización científica, dejando al margen, entre otros, contenidos propios de cada país o localidad, la educación ética y moral, la educación para la ciudadanía y los Derechos Humanos, la educación ambiental, la educación para los medios de comunicación, la educación artística, etc. (Torres Santomé, 2011). Tanto los contenidos demandados como los que no lo son, en la que tal vez es la prueba de evaluación educativa con más capacidad de influencia a nivel internacional, dan pistas sobre la subordinación cultural del ser. Así, diferentes sistemas educativos en el mundo basados en este tipo de lógicas difícilmente escapan a la tendencia mercantilizadora contemporánea (Ball, 
2005), pues bajo la lógica neoliberal la esfera educativa es vista como otra forma para generar beneficios y cultura relacionada con el tener, por su capacidad aculturadora.

\section{¿Tiene que ser así?}

La reflexión sobre cómo son las vías a través de las cuales la cultura del tener se impone sobre la del ser facilita la visualización de una serie de consecuencias que esta imposición genera. Esa lógica del tener ha sido adoptada y reforzada, especialmente en contextos capitalistas, por élites socioeconómicas que basaron en ella la predominancia de su estatus y cultura. Para que esta tendencia ideológica se convierta en pensamiento hegemónico, único, además del tradicional uso directo de la fuerza, se utilizan muchos otros mecanismos que aseguren el sometimiento "consentido" de las alternativas a la misma, a menudo no tan explícitos pero igualmente efectivos, y que en nuestro actual contexto temporal están especialemente basados en la influencia ejercida por medios de comunicación muy poco plurales en cuanto a su posesión. Esta concentración de los medios en manos de pocos grupos empresariales, unida al paulatino debilitamiento de alternativas público-sociales, genera una oferta informativa acorde con ese desequilibrio de fuerzas, y por tanto fuertemente basada en modelos vitales de pensamiento que parten de ideas como la posesión y el consumo, que cumplen con lo que el aparato cultural neoliberal considera como adecuado. Así, la educación, como parte esencial de los sistemas culturales, puede alimentar y contribuir a reproducir la lógica del tener, sumándose sin reflexión interna a demandas productivistas y de inmediatez en resultados enfocados hacia la generación o consumo de recursos materiales, por ejemplo, dejando en segundo plano necesidades conectadas con aspectos más amplios y heterogéneos de la vida, del ser. Pero es imprescindible repetir y recordar que la educación también puede ser un terreno propicio para disputar el dominio de la cultura de la propiedad y apostar hacia una formación cultural más diversa que recoja la variabilidad interna y externa de los seres humanos. 


\section{Referencias bibliográficas}

AMADEU DA SILVEIRA, S. (2009). Novas dimensões da política: protocolos e códigos na esfera pública interconectada. Revista de Sociologia e Politica. 17(34), 103-113.

APPLE, M. (2002). Educar "como dios manda". Mercados, niveles, religión y desigualdad. Barcelona: Paidós.

ARENDT, H. (1998). Los orígenes del totalitarismo. Madrid: Taurus.

BAKAN, J. (2004). La Corporación: la búsqueda patológica de lucro y poder. Barcelona: Volter (Robinbook).

BALL, S. (2005). Educação à venda. Discursos-Cadernos de Política Educativa e Curricular, volume C. Viseu: Livraria Pretexto Editora.

BAUMAN, Z. (2006). Vida Líquida. Barcelona: Paidós.

BECK, U. y BECK-GERNSHEIM, E. (2003). La individualizacion: el individualismo institucionalizado y sus consecuencias sociales y políticas. Bacelona: Paidós Ibérica.

CAMPOS FREIRE, F. (2007). Los principales grupos mundiales de medios de comunicación siguen siendo negocios de familia. Revista Zer, 22, 141-166.

CHOMSKY, N. y HERMAN, E. S. (1990). Los guardianes de la libertad. Barcelona: Crítica. CHOMSKY, N. (1999). El beneficio es lo que cuenta. Barcelona: Crítica.

DIJK, T. A. V. (1999). Ideología. Una aproximación multidisciplinaria. Barcelona: Gedisa.

EMA, J. E. (2013). ¿Qué habrá sido de las ciencias sociales?. Educatio Siglo XXI. 31(2), 15-34.

FOUCAULT, M. (1988). El sujeto y el poder. Revista Mexicana de Sociología. 50(3), 3-20.

FRIEDMAN, M. (2012). Capitalismo y Libertad. Madrid: Síntesis.

FROMM, E. (2007). Del tener al ser. Barcelona: Paidós.

GIROUX, H. (2005). Estudios culturales, pedagogía crítica y democracia radical. Madrid: Editorial Popular.

GRAMSCI, A. (2010). Cartas desde la cárcel. Madrid: Veintisiete Letras.

HILL, D. (2003). O Neoliberalismo Global, A Resistência e a Deformação da Educação. Currículo sem Fronteiras. 3(2), 24-59.

KINCHELOE, J. y STEINBERG, S. (1999). Repensar el multiculturalismo. Barcelona: Octaedro.

KLEIN, N. (2000). No logo. El poder de las marcas. Barcelona: Paidós. (2010). La doctrina del shock. Barcelona: Paidós.

LAVAL, C. (2004). La escuela no es una empresa. Barcelona: Paidós. (2013). La nueva razón del mundo. Ensayo sobre la sociedad neoliberal. Barcelona: Gedisa.

LOCKE, J. (1995). Segundo Tratado sobre el Gobierno Civil. Barcelona: Altaya.

MAX NEEF, M. y SMITH, P. B. (2014). La economía desenmascarada. Barcelona: Icaria.

MOLNAR, Á. (1999). The Commercial Transformation of American Public Education. Ohio Valley Philosophy of Education Conference. Bergamo, Ohio.

MOLNAR, Á. y BONINGER, F. (2007). Adrift: Schools in a total marketing enviroment. The tenth annual report on schoolhouse commercialism trends. Commercialism in Education Research Unit. Tempe: Arizona State University. 
NUSSBAUM, M. (2001). El cultivo de la humanidad. Una defensa clásica de la reforma en la educación liberal. Barcelona: Andrés Bello.

(2006). Las fronteras de la justicia. Barcelona: Paidós.

RAMONET, I. (1995). Pensamiento único y nuevos amos del mundo. En CHOMSKY, N. y RAMONET, I. Cómo nos venden la moto. Barcelona: Icaria.

RAWLS, J. (2002). La Justicia como Equidad. Madrid: Tecnos.

RIÁDIGOS MOSQUERA, C. (2015). Justicia social y educación democrática: un camino compartido. Madrid: La Muralla.

SANTOS, B. de S. (2007). Para além do Pensamento Abissal: Das linhas globais a uma ecologia de saberes. Revista Crítica de Ciências Sociais, 78, 3-46.

SCANLON, T. M. (2003): Lo que nos debemos unos a otros. ¿Qué significa ser moral?. Barcelona: Paidós.

SCHOR, J. B. (2006). Nacidos para comprar. Los nuevos consumidores infantiles. Barcelona: Paidós.

SEN, A. (2010). La idea de la justicia. Madrid: Taurus.

SERRANO, P. (2012). Traficantes de información. La historia oculta de los grupos de comunicación españoles. Madrid: Foca.

SMITH, A. (1776). Investigación sobre la naturaleza y causas de la riqueza de las naciones.

TIMSIT, S. (2002). Stratégies de manipulation.

Recuperado de: http://www.syti.net/Manipulations.html

TORRES SANTOMÉ, J. (2009). La desmotivación del profesorado. Madrid: Morata. (2011). La justicia curricular. El caballo de Troya de la cultura escolar. Madrid: Morata. 
